\title{
Negligência no autocuidado em saúde: a imunização contra a hepatite B na Odontologia
}

\author{
Negligence in health self-care: immunization against hepatitis B in Dentistry \\ El auto-cuidado de la salud en negligencia: \\ la inmunización contra la hepatitis $B$ en Odontología de caso \\ Artênio José Isper GARBIN ${ }^{1}$ \\ Bruno WAKAYAMA ${ }^{2}$ \\ Cléa Adas Saliba GARBIN ${ }^{3}$
}

\begin{abstract}
${ }^{I}$ Professor Adjunto do Departamento de Odontologia Infantil e Social, Faculdade de Odontologia de Araçatuba- UNESP ${ }^{2}$ Mestrando do Programa de Odontologia Preventiva e Social, Faculdade de Odontologia de Araçatuba - UNESP ${ }^{3}$ Professora Titular do Departamento de Odontologia Infantil e Social, Faculdade de Odontologia de Araçatuba- UNESP
\end{abstract}

\begin{abstract}
Resumo
A hepatite B é considerada como um dos principais problemas na saúde pública, devido sua alta virulência e cronicidade em escalas mundiais. Os profissionais da saúde, incluindo os cirurgiões-dentistas, tornam-se vulneráveis aos riscos de infecção a essa doença, devido sua exposição às atividades ocupacionais, assim como, pelos riscos de acidentes com perfurocortantes. Dessa maneira, medidas protetivas, como a imunização contra a hepatite B, são consideradas além de uma prerrogativa profissional, uma atitude de autocuidado em saúde. O objetivo do estudo foi realizar uma revisão de literatura sobre a hepatite $\mathrm{B}$, enfatizando a problemática "A imunização dos cirurgiões-dentistas contra o vírus da hepatite B e seus fatores associados". Para a coleta dos estudos científicos, foram consultadas as bases de dados: Pubmed, Scielo, Bireme e Medline, sem restrição de data e língua. Para a seleção dos estudos, foi realizada a leitura prévia dos títulos e eleitos aqueles a partir dos critérios de inclusão e exclusão. Fizeram parte da revisão 16 artigos datados de 1978 a 2015, com a população do estudo composta por cirurgiões-dentistas, e tipo de intervenção realizada no conhecimento e atitude desses profissionais frente ao tema imunização. Verificou-se que a cobertura vacinal e imunização contra o vírus da hepatite B ainda é uma atitude deficiente entre os cirurgiões-dentistas. Os fatores associados a essa problemática, devem ser discutidos em estudos futuros, dada sua importância nas implicações clínicas odontológicas. Além disso, o conscientizar e a valoração do autocuidado em saúde são pontos essenciais que devem ser considerados para uma atividade profissional responsável.
\end{abstract}

Descritores: Odontologia; Odontólogos: Vacina contra Hepatite B; Imunização.

\begin{abstract}
Hepatitis B is considered a main problem in public health because of its high virulence and chronicity in global scales. Health professionals, including dentists, they are vulnerable to the risk of infection to the disease, due to exposure to occupational activities, as well as the risk of needlestick injuries. Thus, protective measures, as immunization against hepatitis B, are considered as well as a professional prerogative a self-care attitude in health. The aim of the study was to conduct a literature review on hepatitis B, emphasizing the problematic "Immunization of dentists against hepatitis B and its associated factors". For the collection of scientific studies, the databases were consulted: Pubmed, Scielo, Bireme and Medline, no date restriction and language. For the selection of studies, prior reading of titles and those elected from the inclusion and exclusion criteria was performed. They were part of the review 16 articles dated from 1978 to 2015, with the study population comprised of dentists and type of intervention on knowledge and attitude professionals about the immunization theme. It was found that vaccinattion coverage and immunization against hepatitis B virus is still poor attitude among dentists. The factors associated with this problem should be addressed in future studies, given its importance in dental clinical implications. In this way, the awareness and the valuing of health self-care are essential points that should be considered for responsible professional activity.
\end{abstract}

Descriptors: Dentistry; Dentists; Hepatitis B Vaccines; Immunization.

\section{Resumen}

La hepatitis B se considera un problema importante en la salud pública debido a su alta virulencia y cronicidad en escalas globales. Profesionales de la salud, incluidos los dentistas, se vuelven vulnerables al riesgo de infección a la enfermedad, debido a su exposición a las actividades profesionales, así como el riesgo de lesiones com cortopunzantes. Por lo tanto, las medidas de protección, tales como la inmunización contra la hepatitis B, se consideran como una prerrogativa profesional una actitud de auto-cuidado de la salud. El objetivo del estudio fue realizar una revisión de la literatura sobre la hepatitis B, haciendo hincapié en la problemática "La inmunización de los dentistas contra la hepatitis B y sus factores asociados ". Para la seleccíon de los estudios científicos, se consultaron las bases de datos: Pubmed, Scielo, Bireme y Medline, sin restricción de fecha y el lenguaje. Para la selección de los estudios, se realizó la lectura previa de los títulos y los elegidos a partir de los criterios de inclusión y exclusión. Eran parte de la revisión 16 artículos entre 1978 y 2015 , con la población de estudio compuesto por dentistas y tipo de intervención en el conocimiento y la actitud de estos profesionales sobre el tema inmunización. Se encontró que la cobertura de vacunación y la inmunización contra el virus de la hepatitis B sigue siendo mala actitud entre los dentistas . Los factores asociados con este problema deben abordarse en estudios futuros, dada su importancia en implicaciones clínicas dentales. Por otra parte, el conocimiento y la valoración de auto tratamiento son puntos esenciales que deben ser considerados para la actividad profesional responsable.

Descriptores: Odontólogos; Vacunas contra Hepatitis B; Inmunización. 


\section{INTRODUÇÃO}

A hepatite B é uma das principais causas de morbidade e mortalidade no contexto mundial, sendo seus dados epidemiológicos, de grande preocupação pelos principais centros de saúde, caracterizando-a como um problema de saúde pública. Em função disto, começaram a ser pautadas medidas de prevenção e proteção contra $o$ vírus da hepatite $B$ (VHB) a fim de diminuir essas estatísticas, bem como, os óbitos oriundos dessa doença ${ }^{1}$.

A vacina é a principal forma de proteção contra o VHB devido sua segurança, eficiência e grande cobertura populacional. As primeiras vacinas começaram a ser produzidas no início da década 70 e foram aperfeiçoadas com o passar dos anos utilizando tecnologias mais sofisticadas e complexas, a fim de combater essa doença com uma maior eficácia ${ }^{2}$. Disponíveis a partir de 1982, a vacina começou a ser recomendada aos profissionais da saúde nos anos 90 , dada a importância da imunização a esses indivíduos, que estão em contato direto com os principais meios de contaminação e infecção pelo $\mathrm{VHB}^{3}$.

Neste contexto, o cirurgião-dentista é considerado alvo para as doenças hepáticas, devido sua rotina clínica estar intrinsecamente relacionada ao sangue e saliva, que são as principais vias de infecção ao VHB. Além disso, o manuseio de instrumentais e materiais perfurocortantes potencializam os riscos de acidentes comumente relatados nas atividades odontológicas ${ }^{4,5}$.

A verificação da imunização contra o vírus, após as três doses da vacina, é de grande importância para a proteção do profissional a essa doença, pois essa medida de autocuidado só será efetiva após o teste de soroconversão anti-HBS, que irá comprovar de fato a sua imunidade ${ }^{6}$.

Dada a dimensão e relevância da imunização como uma medida protetiva do profissional com a própria saúde, assim como, para a preservação da integridade do paciente, objetivo deste estudo foi elaborar de uma revisão de literatura sobre os principais estudos que abordam o tema imunização dos cirurgiões-dentistas contra o vírus da hepatite B e discutir seus possíveis fatores associados.

\section{MATERIAL E MÉTODO}

Trata-se de uma revisão de literatura, cujo propósito foi discutir as evidências científicas relacionadas à "A imunização dos cirurgiões-dentistas contra o vírus da hepatite B e seus fatores associados".

Para a realização da coleta dos estudos científicos, foi feito a busca nas principais bases de dados: Pubmed, Scielo, Bireme e Mediline. Para a estratégia de busca, foram utilizados os descritores: Odontologia (Dentistry), Odontólogo (Dentist), Vacina contra Hepatite B (Hepatitis B Vacines) e
Imunização (Imunization). Na ordenação da pesquisa dos estudos, foram empregados os Operadores booleanos "AND" e "OR" associados aos descritores para a adequação da estratégia de busca em função do tema a ser explorado.

Após a coleta dos dados, foi feita a leitura dos títulos dos artigos científicos e como critério de exclusão, foram descartados: aqueles que não se adequavam ao tema do estudo e que apresentavam sujeitos diferentes ao delineado. Na segunda fase da análise dos dados, realizou-se a leitura dos resumos dos estudos previamente selecionados, e foram incluídos aqueles que apresentavam o texto completo na íntegra em inglês, português ou espanhol e com a intervenção realizada com base no conhecimento ou atitude frente à imunização contra o vírus da hepatite B.

Foram selecionados 16 artigos científicos publicados entre os anos de 1978 a 2015, com o desenho do estudo do tipo epidemiológico transversal e caso controle (Quadro 1).

\begin{tabular}{|c|c|c|c|c|}
\hline $\mathbf{N}$ & ANO & TÍTULO & SUJEITOS DO ESTUDO & $\begin{array}{l}\text { TIPO DE } \\
\text { ESTUDO }\end{array}$ \\
\hline 1 & 1978 & $\begin{array}{l}\text { Hepatitis and hepatitis } \\
\text { B surface antigen and antibody } \\
\text { in dentists. }\end{array}$ & 414 Cirurgiões-Dentistas & $\begin{array}{l}\text { Exploratório } \\
\text { Transversal }\end{array}$ \\
\hline 2 & 1989 & $\begin{array}{l}\text { Awareness and acceptance of } \\
\text { the hepatitis B vaccine by } \\
\text { dental practitioners in } \\
\text { Malaysia. }\end{array}$ & 730 Cirurgiões-Dentistas & \begin{tabular}{|l} 
Exploratório \\
Transversal
\end{tabular} \\
\hline 3 & 1990 & $\begin{array}{c}\text { Increasing acceptance of } \\
\text { hepatitis B vaccine by dental } \\
\text { personnel but reluctance to } \\
\text { accept hepatitis B carrier } \\
\text { patients. }\end{array}$ & 148 Cirurgiões-Dentistas & \begin{tabular}{|l} 
Exploratório \\
Transversal
\end{tabular} \\
\hline 4 & 1991 & $\begin{array}{l}\text { Hepatitis B vaccination and } \\
\text { infection control in Brazilian } \\
\text { dental practice, } 1990 .\end{array}$ & 947 Cirurgiões-Dentistas & \begin{tabular}{|l} 
Exploratório \\
Transversal
\end{tabular} \\
\hline 5 & 1991 & $\begin{array}{l}\text { Awareness and attitudes } \\
\text { toward hepatitis B among } \\
\text { Malaysian dentists. }\end{array}$ & 1217 Cirurgiões-Dentistas & \begin{tabular}{|l} 
Exploratório \\
Transversal
\end{tabular} \\
\hline 6 & 1994 & $\begin{array}{c}\text { Factors associated with } \\
\text { hepatitis B vaccine response } \\
\text { among dentists. }\end{array}$ & 507 Cirurgiões-Dentistas & \begin{tabular}{|l|} 
Caso \\
controle
\end{tabular} \\
\hline $\begin{array}{llll}7 & & & \\
\end{array}$ & 1999 & $\begin{array}{c}\text { Hepatitis B awareness and } \\
\text { attitudes } \\
\text { amongst dental health care } \\
\text { workers in Riyadh, Saudi } \\
\text { Arabia. }\end{array}$ & 126 Cirurgiões-Dentistas & \begin{tabular}{|l} 
Exploratório \\
Transversal
\end{tabular} \\
\hline 8 & 2003 & $\begin{array}{l}\text { Vacinação contra a hepatite B } \\
\text { entre cirurgiões dentistas }\end{array}$ & 299 Cirurgiões-Dentistas & $\begin{array}{l}\text { Exploratório } \\
\text { Transversal }\end{array}$ \\
\hline 9 & 2008 & $\begin{array}{c}\text { Serological markers and risk } \\
\text { factors related to hepatitis } B \\
\text { virus in dentists in the Central } \\
\text { West region of Brazil }\end{array}$ & 680 Cirurgiões-Dentistas & \begin{tabular}{|l} 
Exploratório \\
Transversal
\end{tabular} \\
\hline 10 & 2008 & $\begin{array}{l}\text { Survey of the level of anti-HBs } \\
\text { antibody titer in vaccinated } \\
\text { Iranian general dentists. }\end{array}$ & 861 Cirurgiões-Dentistas & $\begin{array}{l}\text { Exploratório } \\
\text { Transversal }\end{array}$ \\
\hline 11 & 2010 & $\begin{array}{l}\text { Concerns regarding hepatitis B } \\
\text { vaccination and post- } \\
\text { vaccination test among } \\
\text { Brazilian dentists (discussão) }\end{array}$ & 1302 Cirurgiões-Dentistas & \begin{tabular}{|l} 
Exploratório \\
Transversal
\end{tabular} \\
\hline 12 & 2011 & $\begin{array}{l}\text { Percutaneous injuries } \\
\text { and hepatitis } \\
\text { B vaccination among } \\
\text { Lithuanian dentists. }\end{array}$ & 2235 Cirurgiões-Dentistas & $\begin{array}{l}\text { Exploratório } \\
\text { Transversal }\end{array}$ \\
\hline 13 & 2012 & $\begin{array}{l}\text { Hepatitis-B Vaccination Status } \\
\text { Among Dental Surgeons in } \\
\text { Benin City, Nigeria }\end{array}$ & 70 Cirurgiões-Dentistas & \begin{tabular}{|l} 
Exploratório \\
Transversal
\end{tabular} \\
\hline 14 & 2012 & $\begin{array}{c}\text { Assessment of hepatitis } \\
\text { B vaccination and compliance } \\
\text { with infection control among } \\
\text { dentists in Saudi Arabia. }\end{array}$ & 402 Cirurgiões-Dentistas & \begin{tabular}{|l} 
Exploratório \\
Transversal
\end{tabular} \\
\hline 15 & 2012 & $\begin{array}{l}\text { Vacinação contra hepatite B e } \\
\text { fatores associados entre } \\
\text { Cirurgiōes-Dentistas }\end{array}$ & 333 Cirurgiões-Dentistas & \begin{tabular}{|l} 
Exploratório \\
Transversal
\end{tabular} \\
\hline 16 & 2015 & $\begin{array}{l}\text { HBV Vaccination Status and } \\
\text { Response to Hepatitis B } \\
\text { Vaccine Among Iranian } \\
\text { Dentists, Correlation With Risk }\end{array}$ & 1300 Cirurgiões-Dentistas & \begin{tabular}{|l} 
Exploratório \\
Transversal
\end{tabular} \\
\hline
\end{tabular}

\section{REVISÃO DA LITERATURA}

Estudos relacionados à vacinação contra a hepatite B começaram a ser evidentes nas bases 
científicas, desde que essa doença começou apresentar índices pandêmicos de morbidade e mortalidade em decorrência de um vírus altamente infeccioso e patogênico $^{7}$. Assim sendo, publicação cientifica envolvendo questões relacionadas à aceitação dos cirurgiões-dentistas à vacina contra o VHB foram discussões de estudos epidemiológicos ${ }^{8,9}$.

Berris et $\mathrm{al}^{10}$, no final da década de 70 , já enfatizavam a importância da soroconversão ao vírus da hepatite $B$, verificando os antígenos de superfície da hepatite B (HBsAg) e os anticorpos (Anti-HBS) presentes no plasma sanguíneo dos profissionais da odontologia. Desse modo, estudos nas décadas seguintes foram realizados com o mesmo delineamento metodológico, com o intuito de averiguar a imunização dos cirurgiões-dentistas frente ao VHB. Cleveland et $\mathrm{al}^{11}$ e Paiva et $\mathrm{al}^{12}$, discorreram em seus estudos a relevância do esquema vacinal com as três doses completas para a efetividade da soroconversão, e reiteraram a importância da cobertura vacinal do cirurgião-dentista, dada a atividade ocupacional exercida.

Estudos realizados por Monemi et $\mathrm{al}^{6}$ e Alavian et $\mathrm{al}^{13}$, cujo propósito era verificar a imunização dos cirurgiões-dentistas ao vírus da hepatite $B$, comprovaram por meio de ensaios laboratoriais imunossorventes e imunoenzimáticos, que 88,5\% e $77,3 \%$ dos cirurgiões dentistas, respectivamente, estavam imunes ao vírus da hepatite B com a contagem dos anticorpos anti-HBs $\geq 100 \mathrm{UI} / \mathrm{L}$.

A Organização Mundial da Saúde (OMS), em decorrência dos alarmantes índices epidemiológicos das doenças hepáticas virais, criou diretrizes e recomendações para o combate as hepatites, ressaltando a importância da imunização contra o VHB, principalmente aos grupos vulneráveis, o qual insere-se os cirurgiões-dentistas ${ }^{14}$.

Com a introdução dessas medidas protetivas em saúde, houve receios por parte dos profissionais da odontologia em aderir tais recomendações de autocuidado. Yaacob et $\mathrm{al}^{8}$ e Azoodo et $\mathrm{al}^{9}$, ao analisar essa questão, verificaram que as principais preocupações em vacinarem-se, estavam relacionadas aos efeitos colaterais; o risco de infecção ao HIV a partir da vacinação; o alto custo e a falta de informação. Neste mesmo período foi relatado por Scully et $\mathrm{al}^{15}$, que muitos profissionais da odontologia tinham aflições e recusavam realizar atendimentos clínicos aos pacientes diagnosticados com a hepatite $\mathrm{B}$, mesmo estando imunes ao vírus.

A utilização de Equipamentos de Proteção Individual e a biossegurança no atendimento clínico são medidas fundamentais para prevenir e minimizar o risco de contaminação por algum tipo de patógeno ${ }^{16}$. Entretanto, Razak et al17 e Martins et al $^{18}$, em seus estudos, constataram que tais medidas de proteção física, por tempo foram negligenciadas pelos profissionais da odontologia, principalmente, por aqueles que não estavam vacinados contra o vírus da hepatite $B$ ou que apresentavam a vacinação incompleta, aumentando então, o riscos de infecções cruzadas da hepatite B.

Em relação aos acidentes com perfurocortantes e a situação vacinal, autores Al-Dharrab et $\mathrm{al}^{19}$, Rimkuviene et $\mathrm{al}^{20}$ ratificaram a importância da cobertura vacinal aos cirurgiões-dentistas devido as elevadas prevalências de acidentes ocupacionais decorrentes da profissão. Da mesma maneira, Almeida et $\mathrm{al}^{21}$, Paul et $\mathrm{al}^{22}$ discutem a importância do fortalecimento de políticas públicas voltadas a essa questão, a fim de orientar e conscientizar os profissionais sobre a necessidade da imunização como uma ferramenta de proteção a saúde.

\section{DISCUSSÃO}

As publicações científicas incluídas nesta revisão, mesmo sendo realizadas em décadas e nacionalidades distintas, apresentam objetivos e delineamentos semelhantes ao avaliar o estado de imunização dos cirurgiões-dentistas ao vírus da hepatite $\mathrm{B}$, tal como, sua relação às atitudes e práticas clínicas. Esse ciclo de estudos foi norteado pela perspectiva de verificar, em cada momento, as peculiaridades das políticas públicas voltadas a esse tema, as mudanças de comportamento e as atitudes no autocuidado em saúde após as recomendações da OMS.

No Brasil a atenção voltada à prevenção e proteção contra a hepatite $\mathrm{B}$ é pontuada desde o início dos anos 90, com a vacinação contra o VHB aos grupos vulneráveis, incluindo os profissionais da saúde. Nos anos seguintes, devido à dimensão da doença e a necessidade de implantação de politicas públicas que viabilizassem de forma universal, integral e com equidade o acesso da população aos serviços que versavam sobre as hepatites virais, foi instituída pela Portaria $\mathrm{n}^{\circ}$ 2.080, o Programa Nacional para a Prevenção e o Controle das Hepatites Virais $\mathrm{PNHV}^{14}$.

Dada essa importância, os estudos nacionais ${ }^{12,18,23}$ incluídos nessa revisão apresentaram prevalências altas em relação a vacinação completa com as três doses, entretanto, somente essa medida não é suficiente para garantir, de fato, a imunização. Dessa maneira, deve-se ressaltar a verificação da cobertura vacinal com a realização do teste anti-HBS para comprovar, a validade da sua cobertura vacinal contra o vírus $^{23}$.

$\mathrm{O}$ receio dos profissionais da odontologia no final da década de 80 em receberem as vacinas contra $\mathrm{o} \mathrm{VHB}^{8}$, muito está relacionado às formas e tecnologias em que eram utilizadas para sua 
fabricação. As primeiras vacinas criadas foram originadas do plasma sanguíneo purificado de pessoas com hepatite B assintomática, estabelecendo então, margens de dúvidas e inseguranças quanto à efetividade, a dimensão dos efeitos colaterais e a coinfecção pelo HIV.

No entanto, nos dias atuais, com a evolução genética e tecnológica, as vacinas são produzidas em maiores contingencias, sendo mais seguras e imunogênicas. Além disso, é comprovado cientificamente a sua eficácia na proteção ao VHB e são amplamente distribuídas, tornando mais fácil o acesso à imunização colaborando para a redução das taxas de cronicidade da doença ${ }^{2}$.

Os fatores associados, em relação ao tema abordado nas produções científicas analisadas nessa revisão, evidenciam lacunas a serem discutidas, em detrimento dos riscos e agravos que podem repercutir a saúde dos cirurgiões-dentistas, devido suas atividades ocupacionais. Considerando tal explanação, Razak et $\mathrm{al}^{17}$ e Martins et $\mathrm{al}^{18}$, em seus estudos comprovaram negligências dos profissionais não vacinados ou com o esquema vacinal incompleto, quanto a utilização de equipamentos de proteção individual. Embora os períodos de publicação sejam distintos, a problematização ainda se fez presente em ambas às situações, determinando assim, a relevância da criação de medidas educacionais voltadas para a prática de biossegurança nos consultórios odontológico de forma mais conscienciosa e crítica ${ }^{24}$.

Nas questões envolvendo os acidentes com perfurocortantes, grande parte dos autores relatou ocorrência desse incidente em algum momento da atividade profissional dos sujeitos do estudo. De forma geral, a atividade laboral exercida pelo cirurgião-dentista já é um fator de risco para os acidentes, entretanto, condutas clínicas conscientes e prudentes são essenciais para a prevenção desses imprevistos. Além disso, fatores como o correto descarte de Resíduos de Serviço de Saúde $^{25}$ e o não reencape de agulhas ${ }^{26}$ (maior prevalência de acidente) devem questões pontuadas na formação e reciclagem profissional dos cirurgiões-dentistas.

A problematização da imunização contra o vírus da hepatite B, ainda é um obstáculo a ser confrontado pela saúde pública e suas esferas operacionais, visto que, suas estimativas ainda são desproporcionais diante das ações preventivas que são realizadas ${ }^{3}$.

$\mathrm{O}$ desconhecimento e a negligência dos cirurgiões-dentistas em verificar sua imunidade com o teste anti-HBS, ainda é relevante, em virtude da dimensão dos riscos de infecção pelo vírus, assim como, por ser a principal forma de reduzir os índices de portadores crônicos da hepatite e minimizar a disseminação da doença ${ }^{5}$.

\section{CONCLUSÃO}

A imunização contra o vírus da hepatite $\mathrm{B}$ e a verificação da sua cobertura vacinal, ainda não é retratada como uma realidade unanime e efetiva entre cirurgiões-dentistas. Os fatores associados a essa problemática, devem ser discutidos em estudos futuros, dada sua importância nas implicações clínicas odontológicas.

A motivação e a sensibilização à vacinação é uma das principais formas de adesão dos profissionais as medidas de autocuidado. $O$ conscientizar e a valoração da própria saúde são pontos essenciais a ser considerado para uma atividade profissional responsável.

\section{REFERÊNCIAS}

1. Souza FO, Freitas PSP, Araújo TM, Gomes MR. Vacinação contra hepatite B e Anti-HBS entre trabalhadores da saúde. Cad. Saúde Colet. 2015;23(2):172-9.

2. Moraes JC, Luna EJ, Grimaldi RA. Immunogenicity of the Brazilian hepatitis B vaccine in adults. Rev Saude Publica. 2010;44(2):353-9

3. Resende VLS; Abreu MHNG; Teixiera R; Pordeus IA. Hepatites Virais na Prática Odontológica: Riscos e Prevenção. Pesq Bras Odontoped Clin Integr. 2010;10(2):317-23.

4. Garbin CAS, Martins RJ, Garbin AJI, Hidalgo LRC. Conductas de estudiantes del área de la salud frente a la exposición ocupacional a material biológico. Cienc Trab. 2009;11(31):18-21.

5. Ferreira, RC, Guimarães, ALS, Pereira RD, Andrade RM, Xavier RP, Martins AMEDB. Vacinação contra hepatite $\mathrm{B}$ e fatores associados entre cirurgiões-dentistas. Rev Bras Epidemiol. 2012;15(2):315-23.

6. Momeni N, Ahmad Akhoundi MSA, Alavian SM, Shamshiri AR, Norouzi M, Mahboobi N, Moosavi $\mathrm{N}$, Jazayeri SM. HBV Vaccination status and response to hepatitis $b$ vaccine among iranian dentists, correlation with risk factors and preventive measures. Hepat Mon 2015;15(1):e20014.

7. Milani RM, Canini SRMS, Garbin LM, Teles SA, Gir E, Pimenta FR. Imunização contra hepatite B em profissionais e estudantes da área da saúde: revisão integrativa. Rev Eletr Enf. 2011;13(2):323-30.

8. Yaacob HB, Samaranayake LP. Awareness and acceptance of the hepatitis B vaccine by dental practitioners in Malaysia. $\mathrm{J}$ Oral Pathol Med. 1989;18(4):236-9.

9. Azodo C, Ehizele A, Uche I, Erhabor P. Hepatitis$B$ vaccination status among dental surgeons in 
benin city, Nigeria. Ann Med Health Sci Res. 2012;2(1):24-8.

10. Berris B, Feinman SV, Sinclair JC, Wrobel D. Hepatitis and hepatitis B surface antigen and antibody in dentists. Can Med Assoc J. 1978;119(9):1040-3.

11. Cleveland JL, Siew C, Lockwood SA, Gruninger SE, Chang SB, Neidle EA, Russell CM. Factors Associated with Hepatitis B Vaccine Response Among Dentists. J Dent Res.1994;73(5):1029-35.

12. Pavia EMM, Triplle AFV, Silva EP, Cardoso DDP. Serological markers and risk factors related to hepatitis B virus in dentists in the Central West region of Brazil. Braz J Microbiol. 2008;39(2):251-6.

13. Alavian SM, Izadi M, Zare AA, Lankarani MM, Assari S, Vardi MM. Survey of the level of anti-HBs antibody titer in vaccinated Iranian general dentists. Spec Care Dentist. 2008;28(6):265-70.

14. Brasil. Ministério da Saúde. Secretaria de Vigilância em Saúde. Departamento de DST, Aids e Hepatites Virais. Manual A B C D E das Hepatites Virais para Cirurgiões Dentistas / Ministério da Saúde, Secretaria de Vigilância em Saúde, Departamento de DST, Aids e Hepatites Virais. - Brasília: Ministério da Saúde, 2010.

15. Scully C, Pantlin L, Samaranayake LP, Dowell TB. Increasing acceptance of hepatitis B vaccine by dental personnel but reluctance to accept hepatitis B carrierpatients. Oral Surg Oral Med Oral Pathol. 1990;69(1):45-7.

16. Milfont JAC, Oliveira AHA. Equipamentos de proteção individual em odontologia: revisão integrativa de literatura. INTERFACES. 2015; 3(8):1-6.

17. Razak IA, Latifah RJ, Nasruddin J, Esa R. Awareness and attitudes toward hepatitis B among Malaysian dentists. Clin Prev Dent. 1991;13(4):22-4.

18. Martins AMEB, Barreto SM. Vacinação contra a hepatite B entre cirurgiões dentistas. Rev Saúde Pública. 2003;37(3):333-8.

19. Al-Dharrab AA, Al-Samadani KH. Assessment of hepatitis $\mathrm{B}$ vaccination and compliance with infection control among dentists in Saudi Arabia. Saudi Med J. 2012;33(11):1205-10.

20. Rimkuviene J, Puriene A, Peciuliene V, Zaleckas L. Percutaneous injuries and hepatitis B vaccination among Lithuanian dentists. Stomatologija. 2011;13(1):2-7.

21. Almeida OP, Scully C, Jorges J. Hepatitis B vaccination and infection control in Brazilian dental practice, 1990. Community Dent Oral Epidemiol. 1991;19(4):225-7.
22. Paul T, Maktabi A, Almas K, Saeed S. Hepatitis B awareness and attitudes amongst dental health care workers in Riyadh, Saudi Arabia. Odontostomolatol Trop. 1999;22(86):9-12.

23. Resende VLS, Abreu MHG, Paiva SM, Teixeira $\mathrm{R}$, Pordeus IA. Concerns regarding hepatitis B vaccination and post-vaccination test among Brazilian dentists. Virol J. 2010;7:154.

24. Garbin CAS, Garbin AJI, Saliba TA, Bino LS, Parisoto GB. Avaliação do conhecimento dos estudantes de odontologia da FAI - Faculdades Adamantinenses Integradas sobre normas de biossegurança. Rev OMNIA SAÚDE. 2007;4(1):17-22.

25. Garbin AJI, Wakayama B, Brito CP, Garbin CAS. A imperícia no gerenciamento dos Resíduos de Serviços de Saúde nos consultórios odontológicos privados. Arch Health Invest. 2015;4(5):1-5.

26. Martins RJ, Moimaz SAS, Sundefeld, MLMM, Garbin, AJI, GONÇAVES PRV, Garbin CAS. Adesão às precauções padrão sob o prisma do Modelo de Crenças em Saúde: a prática de reencapar agulhas. Ciênc saúde coletiva. 2015:20(1):193-8.

\section{CONFLITO DE INTERESSES}

Os autores declaram não haver conflitos de interesse.

\section{AUTOR PARA CORRESPONDÊNCIA}

\section{Bruno Wakayama}

brunowakayama@gmail.com 